\title{
Prediction of the Blood-Brain Barrier Permeability Using RP-18 Thin Layer Chromatography
}

https://doi.org/10.1515/chem-2019-0005

received July 13, 2018; accepted November 2, 2018.

\begin{abstract}
The Blood-Brain Barrier (BBB) permeability is an important factor governing a drug's ability to act upon the Central Nervous System. The measure of the BBB permeability used throughout this study is the log BB (the blood/brain partitioning coefficient) measured in vivo or calculated. Useful yet simple models of the BBB permeability were developed by Stepwise Multiple Regression Analysis based on the chromatographic parameters $\mathbf{R}_{\mathbf{f}}$ and $\mathbf{R}_{\mathrm{f}} /$ PSA obtained by RP-18 TLC with acetonitrile - pH 7.4 phosphate buffered saline 70:30 $(\mathrm{v} / \mathrm{v})$ as mobile phase, combined with descriptors - the number of $\mathrm{H}$-bond donors (HD), the number of $\mathrm{H}$-bond acceptors (HA), energy of the highest occupied molecular orbital - (eH), energy of the lowest unoccupied molecular orbital (eL). The ability of the solutes to cross the BBB has been studied qualitatively using Discriminant Function Analysis. Almost all compounds with the known BB vivo parameter were correctly classified as CNS+/-. The classification functions based on $\mathbf{R}_{\mathrm{f}} / \mathbf{P S A}$ have been verified using an external group. The results of the chromatographic analysis proposed in this study (RP-18 TLC) are a source of valuable information on the BBB permeability of compounds available even on a very small scale.
\end{abstract}

Keywords: RP-18 TLC; QSAR; Blood-Brain Barrier; chemometric techniques.

\footnotetext{
*Corresponding author: Anna W. Sobańska, Department of Analytical Chemistry, Medical University of Lodz, ul. Muszyńskiego 1, 90-151 Lodz, Poland, E-mail: anna.sobanska@umed.lodz.pl Karolina Wanat, Elżbieta Brzezińska: Department of Analytical Chemistry, Medical University of Lodz, ul. Muszyńskiego 1, 90-151 Lodz, Poland
}

\section{List of Abbreviations}

BBB- Blood-Brain Barrier

BB - Blood/Brain Partition Coefficient

CNS - Central Nervous System

D - Distribution Coefficient

DFA - Discriminant Function Analysis

DM - Total Dipole Moment

eH - Energy of the Highest Occupied Molecular Orbital

eL - Energy of the Lowest Occupied Molecular Orbital

HA - Hydrogen Bond Acceptor

HD- Hydrogen Bond Donor

LMO- Leave-Many-Out

LOO - Leave-One-Out

MRA - Multiple Regression Analysis

Mw - Molecular Weight

NPTLC - Normal-Phase Thin Layer Chromatography

$\mathrm{P}$ - Partition Coefficient

PRESS - Predicted Residual Sum of Squares

PSA - Polar Surface Area

$\mathrm{R}_{\mathrm{f}}$ - Retardation Factor

RPTLC - Reversed-Phase Thin Layer Chromatography

$\mathrm{S}_{\mathrm{a}}$ - Grid Surface Area

SOTLC- Salting-Out Thin Layer Chromatography

SDEP - Standard Deviation of Error of Prediction

TPSA - Topological Polar Surface Area

$\mathrm{V}$ - van der Waals molar volume

\section{Introduction}

The blood-brain barrier (BBB) is the main physical and enzymatic barrier that separates brain tissues and blood, prevents the penetration of the central nervous system (CNS) by undesired substances (peripherally acting drugs or harmful compounds) and facilitates its penetration by neurotherapeutics [1-3]. The most common measures of the BBB permeability are $\log$ BB - the blood/brain partitioning coefficient at equilibrium conditions, i.e. the ratio of the drug concentration in the brain to that in the blood plasma measured at some defined time point 
and the less frequently used kinetic permeability $\log$ PS, where $\mathrm{P}$ is the observed permeability across $\mathrm{BBB}(\mathrm{cm} / \mathrm{s})$ and $S$ is the surface area of brain capillary endothelium $\left(\mathrm{cm}^{2} / \mathrm{g}\right)[3,4]$. The evaluation of the compound's ability to cross the BBB is a vital step in assessing its therapeutic potential because many promising neurotherapeutics miss their goal due to their inability to enter the brain or, oppositely, peripherally acting drugs exhibit unwanted side effects due to their undesired brain penetration. The in vivo, in vitro and in silico methods used currently to study the drug brain penetration are discussed in a number of review papers $[2,5,6]$. High costs and low throughput of any in vivo experiments as well as the need to use lab animals are the reasons why several in vitro cellbased methods of the BBB permeation assessment have been developed [7]. Concurrently with the development of the in vitro cell-based techniques, some non-cell-based assays have been introduced [8]. As more data on the CNS activity of different compounds were gathered, several attempts were made to generate computational models linking the BBB permeability of compounds to their experimentally measured and/or theoretically calculated physicochemical features [4,5].

It has been known for several years that the lipophilicity of a molecule is an important factor influencing its BBB passage. The relationship between the compound's lipid solubility and its ability to enter the brain was observed as early as in the late nineteenth century [9]. Later studies have focused on correlations between the brain uptake and the lipophilicity of the solutes often expressed as the partition coefficient $\log \mathbf{P}$ between water and certain water-immiscible solvents, of which 1-octanol is the most important example $\left(\log \mathbf{P}_{\text {oct }}\right)$. It was observed that for a congeneric series of compounds of the hypnotic activity the most active ones are those with $\log \mathbf{P}_{\text {oct }}$ around 2 ("the rule of 2") [10]. Further research supported this hypothesis for a series of imidazoles injected intravenously into rats, linking the brain uptake expressed as $\log \left(\boldsymbol{C}_{\text {brain }} / \boldsymbol{C}_{\text {i.v. }}\right)$ with the water-pH 7.4 buffer apparent partition coefficient $\log \mathbf{P}_{\text {oct }}$ via the quadratic equation: $\log \left(C_{\text {brain }} / C_{\text {i.v. }}\right)=-0.94+0.574 \log \mathrm{P}_{\text {oct }}{ }-0.133$ $\left(\log \mathbf{P}_{\text {oct }}\right)^{2}\left(n=14, R^{2}=0.897\right)$ with the maximum (as it can be easily calculated) at $\log \mathbf{P}_{\text {oct }}{ }^{\prime}=2.16$ [11], and for a series of C-11 labelled radiopharmaceuticals [12].

Later studies [13] did not confirm the general nature of the $\log \mathbf{B B}-\log \mathbf{P}_{\text {oct }}$ parabolic relationship. It was soon observed that the octanol-water based lipophilicity is not an ideal parameter to predict the BBB permeation for larger and more diverse groups of compounds. The application of other measures of lipophilicity was proposed of which $\log \mathbf{P}$ in other solvent systems such as cyclohexane-water $\left(\log P_{\text {cyc }}\right)$, chloroform-water $\left(\log P_{\text {chlor }}\right)$ or $\Delta \log P=\log P_{\text {oct }}$ $-\log P_{\text {cyc }}$ seemed promising but in spite of their relatively high predictive value they have not attracted broader attention because only few $\log \mathbf{P}$ values for these systems are available.

Attention was then turned to the partitioning of solutes in other bi-phase systems such as chromatographic systems resembling the properties of biomembranes. Thin layer chromatography is a low-cost, rapid and easy-toperform separation technique whose main application in drug development process is at present the octanol/water lipophilicity determination [14-16]. The most important thin layer chromatographic parameter used to evaluate the lipophilicity is the $\mathbf{R}_{\mathrm{M}}$ value introduced by Bate-Smith and Westall: $\mathbf{R}_{\mathrm{M}}=\log \left(\mathbf{1} / \mathbf{R}_{\mathrm{f}} \mathbf{- 1}\right)$ [17].

Reversed-phase thin layer chromatography is particularly useful for the determination of solutes' partition between water and the biomembrane because the partition between a non-polar sorbent and a purely aqueous mobile phase resembles that between water and the biophase. Because reversed-phase chromatography with the purely aqueous mobile phase is impractical due to very long retention times (or very low $\mathbf{R}_{\mathrm{f}}$ ), the $\mathbf{R}_{\mathrm{M}}{ }^{0}$ values are obtained by extrapolation of plots of $\mathbf{R}_{\mathrm{M}}$ versus the concentration of the organic modifier to zero concentration of the modifier (pure water/buffer). This extrapolation is usually conducted by using the linear Soczewinski-Wachmeister equation (called sometimes the "Soczewiński-Matysik" equation) [18]: $\mathbf{R}_{\mathrm{M}}=\mathbf{R}_{\mathrm{M}}{ }^{0}-\mathbf{S} \boldsymbol{\varphi}$ ( $\varphi$ is the volume fraction of the organic modifier in the mobile phase); some authors suggest that the relationship between $\mathbf{R}_{\mathrm{M}}$ and $\boldsymbol{\varphi}$ should be described with the quadratic equation $\mathbf{R}_{\mathrm{M}}=\mathbf{R}_{\mathrm{M}}{ }^{0}+\mathbf{b} \boldsymbol{\varphi} \boldsymbol{+} \mathbf{a} \boldsymbol{\varphi}^{2}$ [19].

The slope $\mathbf{S}$ of the linear Soczewiński-Wachmeister equation is another useful measure of lipophilicity, related to the hydrophobic surface of a molecule [19]. On the basis of $\mathbf{R}_{\mathrm{M}}{ }^{\mathbf{0}}$ an alternative lipophilicity parameter, $\mathbf{C}_{\mathbf{0}}$, has been proposed. This parameter is calculated according to the equation: $\mathbf{C}_{\mathbf{0}}=\mathbf{R}_{\mathrm{M}}{ }^{\mathbf{}} / \mathbf{S}$ and represents the volume fraction of the organic solvent in the mobile phase for which the amount of the solute in the mobile phase is equal to that in the stationary phase [20].

The RP-thin layer chromatographic descriptors $\mathbf{R}_{\mathrm{M}}{ }^{\mathbf{0}}$ and $\mathbf{S}$ have been used to evaluate the brain penetration of solutes by several authors [21-26]. The relationships between $\log \mathbf{B B}$ and $\mathbf{R}_{\mathrm{M}}{ }^{0}$ obtained by these authors are, generally speaking, linear or quadratic, depending on the group of solutes; the relationships between $\log \mathbf{B B}$ and $\mathbf{S}$ described in Ref. [23] are parabolic.

Although less frequently than by RP TLC, the retention data used as the blood-brain barrier 
permeability predictors may be collected by other planar chromatographic techniques [24,27]. In normal-phase TLC the relationship between $\boldsymbol{\varphi}$ (the volume fraction of the more polar component of the mobile phase) and $\mathbf{R}_{\mathrm{M}}$ can be expressed by the equation: $\mathbf{R}_{\mathrm{M}}=\mathbf{R}_{\mathrm{M}}{ }^{0}-\mathbf{b} \log \boldsymbol{\varphi}$. NP-TLC $\mathbf{R}_{\mathrm{M}}{ }^{\mathbf{0}}$ values can be correlated with $\log \mathbf{B B}$ via the parabolic relationships [27]. Quite recently salting-out thin layer chromatography (SOTLC) - the separation technique involving the application of concentrated solutions of inorganic salts as mobile phases on silicagel, aluminum oxide or cellulose supports [28] - has been used to study the BBB permeability of solutes according to a novel procedure by Ciura et al. [29] utilizing the chromatographic parameter $\mathbf{C}_{\mathbf{0}}=\mathbf{R}_{\mathrm{M}}{ }^{0} / \mathbf{m}$ (derived from the equation $\mathbf{R}_{\mathrm{M}}=\mathbf{R}_{\mathrm{M}}{ }^{0}$ $+\mathbf{m C}$, where $\mathbf{C}$ is the salt molar concentration).

Although lipophilicity is a key factor governing the solutes' ability to cross the BBB and hence the chromatographic lipophilicity descriptors play a dominant role in several BBB permeation models, there are other molecular parameters (related mainly to the molecule's size and polarity) that influence the BBB passage [30]. According to some earlier research, Polar Surface Area (PSA) alone or combined with lipophilicity is an important predictor of the BBB permeability and two useful yet simple models of the blood-brain partitioning were proposed by Norinder $(\log \mathbf{B B}=\mathbf{0 . 1 3 9}+\mathbf{0 . 1 5 2} \log \mathbf{P}$ 0.0148 PSA) [31] and Clark (log BB $=0.547-0.016$ PSA $)$ [32].

Milosevic et al. [24] proposed the multiple regression BBB permeation models involving chromatographic descriptors (RPTLC $\mathbf{R}_{\mathrm{M}}{ }^{0}$ or NPTLC $\mathbf{C}_{\mathbf{0}}$ ) combined with topological polar surface area (TPSA) and according to Ciura [29] the SOTLC $\mathbf{C}_{\mathbf{0}}$ parameter should be combined with several other descriptors, including TPSA.

In our earlier research we created some useful models, capable of the quantitative prediction of the BBB permeability of solutes [33,34] involving the retardation factor $\mathbf{R}_{\mathbf{f}}$ (RP-18 TLC) determined for a single composition of the mobile phase (without the need to extrapolate as in the approaches based on $\mathbf{R}_{\mathrm{M}}{ }^{0}$ or $\mathbf{C}_{\mathbf{0}}$ ). The single chromatographic run approach, although used less frequently than the extrapolation technique, is recommended by some authors for lipophilicity determination [35,36] and the chromatographic data obtained via this approach proved useful in the BBB permeability estimations presented in Ref. [33,34]. The $\mathbf{R}_{\mathrm{f}}$ values were combined with some easily calculable molecular descriptors, selected by stepwise multiple regression analysis (MR) and traditionally linked to good brain penetration $(\log \mathbf{D}$, PSA, the number of hydrogen bond donors and acceptors HD and HA, dipole moment
DM). The same RP-chromatographic descriptors $\left(\mathbf{R}_{\mathrm{f}}, \mathbf{R}_{\mathrm{f}} /\right.$ PSA) were used in our earlier research to study the solutes that cross the BBB (CNS+) and the compounds that do not exhibit good brain penetration (CNS-) [33,34].

Initially the efficiency of our methodology based on a RP 18 TLC model was confirmed for a group of CNSactive drugs [33]; then the applicability of our approach was acknowledged for some sunscreens and excipients in pharmaceutical and cosmetic preparations, whose CNS bioavailability should be limited [34]. In this paper we are proving a high predictive value and a universal applicability of our analytical model for a large group of cases of significant structural diversity and different sites of action.

In our current study we managed to demonstrate that the proposed analytical and mathematical models can be applied to any chemical compound of both CNS+ and CNS- activity. We confirmed the retardation factor $\mathbf{R}_{\mathrm{f}}$ and the chromatography-derived descriptor $\mathbf{R}_{\mathrm{f}} / \mathbf{P S A}$ proposed in our previous paper [33] as universal predictors of good brain absorption and suggested adding them as novel parameters to the list of the currently recognized conditions of the BBB permeability [30].

\section{Experimental}

\subsection{Materials}

154 drugs analyzed during these investigations (Figure 1, Supplementary Materials) were isolated from pharmaceutical preparations $(\underline{1}, \underline{4}, 5,7-10,12-21,23,24$, 26, 28-44, 47-51, 54-69, 71, 73-111, 131, 132, 144-154), purchased from Sigma-Aldrich $(\underline{2}, \underline{3}, \underline{6}, \underline{11}, \underline{22}, \underline{25}, \underline{27}, \underline{52}$, $\underline{53}, \underline{72}, \underline{114})$ or donated as free samples by CIBA (113), PolfaPabianice $(\underline{115}, \underline{116}, \underline{133}-143)$, BASF (무, 120, 121, 127, 129), Merck $(\underline{117}-\underline{119}, \underline{122}, \underline{123}, \underline{126}, \underline{130})$ and Symrise $(\underline{124}, \underline{125}$, 128). The purity of drugs isolated from pharmaceutical preparations was assessed by Thin Layer Chromatography and densitometry (Section 2.2.). All isolated drugs gave single chromatographic spots (densitometric peaks) and were used without further purification. Drugs purchased from Sigma-Aldrich were of analytical or pharmacopeial grade. Distilled water used for chromatography was from an in-house distillation apparatus. Analytical grade acetonitrile and methanol were from Avantor Performance Materials (formerly Polskie Odczynniki Chemiczne). $\mathrm{pH}$ 7.4 phosphate buffered saline was from Sigma-Aldrich. Some of the analyzed compounds (52 cases): $\underline{\mathbf{3}} \mathbf{- 4}, \underline{\mathbf{6}}, \mathbf{9}$, $\underline{11}, \underline{16}, \underline{18-19}, \underline{22}-26, \underline{29}-\underline{33}, \underline{36}-\underline{37}, \underline{40}-\underline{41}, 43-44, \underline{48}-\underline{-50}$, 
52-53, 62, 64- $\underline{66}, \underline{69}, \underline{70}$ and $\underline{114}-\underline{130}$ were investigated chromatographically and presented in previously defined populations [33,34]. The chromatographic data and physicochemical descriptors of these compounds were introduced into the analysis.

\subsection{Thin layer chromatography}

Thin layer chromatography was performed on 10x20 cm glass-backed TLC plates coated with an RP-18 $\mathrm{F}_{254 \mathrm{~s}}$ layer from Merck, Germany (layer thickness $0.25 \mathrm{~mm}$ ). Before use, the plates were pre-washed with methanoldichloromethane 1:1 (v/v) and dried overnight in ambient conditions. Solutions of compounds $\underline{\mathbf{1}} \underline{\mathbf{1 5 4}}$ in methanol $\left(1 \mu \mathrm{g} \cdot \mu \mathrm{L}^{-1}\right.$, spotting volume $\left.1 \mu \mathrm{L}\right)$, were spotted with the Hamilton microsyringe, $15 \mathrm{~mm}$ from the plate bottom edge, starting $10 \mathrm{~mm}$ from the plate edge, at $8 \mathrm{~mm}$ intervals. The chromatographic plates were developed in a vertical chromatographic chamber lined with filter paper and previously saturated with the mobile phase vapor for 20 min. The mobile phase consisted of acetonitrile - $\mathrm{pH} 7.4$ phosphate buffered saline 70:30 (v/v). The development distance was $95 \mathrm{~mm}$ from the plate bottom edge. After development, the plates were dried at room temperature and examined under UV light $(254 \mathrm{~nm})$ and with the Desaga CD60 densitometer (Multiwavelength Scan, 200-300 $\mathrm{nm}$ at $20 \mathrm{~nm}$ intervals). All chromatograms were repeated in duplicate, and the mean $\mathbf{R}_{\mathrm{f}}$ values were used in further investigations.

\subsection{Calculated molecular descriptors}

The molecular descriptors for compounds investigated during this study were calculated with HyperChem 7.0 [37], utilizing PM3 semi-empirical method with Polak-Ribiere'a algorithm [38] (total dipole moment - DM [D], logarithm of the octanol/water partition coefficient - $\log \mathrm{P}$, van der Waals molar volume $-\mathbf{V}(\mathrm{V} / 100)\left[\AA^{3}\right]$, grid surface area $\mathbf{S}_{\mathbf{a}}\left(\right.$ as $\left.\mathrm{S}_{\mathrm{a}} / 100\right)\left[\AA^{2}\right]$, molecular weight - Mw (as $\left.\mathbf{M}_{\mathrm{w}} / 100\right)[\mathrm{g} /$ mol], energy of the highest occupied molecular orbital - eH $[\mathrm{eV}]$, energy of the lowest unoccupied molecular orbital - eL (eLx10) [eV]). The distribution coefficient - $\log$, polar surface area - \%PSA, and PSA $\left[\AA^{2}\right]$, the number of H-bond donors - HD and the number and of H-bond acceptors - HA were calculated using ACD/Labs 8.0 software [39]. The theoretical values describing BBB permeability were calculated $\mathbf{B} 1(\log \mathrm{BB}=0.139+0.152 \log$ $\mathrm{P}-0.0148 \mathrm{PSA})$ [31] and B2 ( $\log \mathrm{BB}=0.547-0.016 \mathrm{PSA})$ [32]
The experimental $\mathrm{BBB}$ permeability (BB vivo) values and CNS+/CNS- binary BBB bioavailability scores were taken from the literature sources [40,41]. The chromatographic data and molecular descriptors for compounds $\mathbf{1}-\mathbf{1 5 4}$ are presented in Tables 1a-e (Supplementary Materials).

\subsection{Statistical analysis}

154 compounds analyzed during these investigations were divided into two subsets: the training set (compounds with the known experimental BBB permeability BB vivo, values $\underline{1}-\underline{46}$ ) and the training set (compounds without the known experimental BBB permeability BB vivo, values 47-154).

\subsubsection{Stepwise Multiple Regression Analysis}

Thephysicochemical parameters related to thecompounds' BBB permeability were determined by the use of MR analysis. The stepwise multiple regression analysis and the correlation analysis were carried out using STATISTICA 10.0 [42]. The values of the BBB permeability (BB vivo), determined for 46 cases, and the calculated values of $\log$ BB (B1 and B2) for 154 analyzed compounds were used as dependent variables and other physicochemical molecular descriptors and chromatographic data as independent variables. The statistical significance ( $p$-level) of a result as an estimated measure of the degree to which it represents the population was determined as $p \leq 0.05$. The correlation matrix was used to correlate the biological activities with the various variables. If two independent variables showed a correlation greater than $R^{2}>0.4$ one of them was removed.

Validation of the correlation models was carried out by the general internal cross-validation procedures: "leaveone-out" ( $L O O)$ and "leave-many-out" ( $L M O)$. In the $L O O$ approach, one element is removed from the whole data set and used to verify the model generated with the remaining $\mathrm{n}-1$ elements; the procedure is then repeated with another element. In the $L M O$ method the data set is repeatedly divided into two subsets used for model generation and its verification, respectively. The predictive power of the developed models was evaluated using the following indicators: cross-validated squared correlation coefficient $\left(Q_{L O(M) O}^{2}\right)$, predicted residual sum of squares (PRESS), standard deviation based on PRESS $\left(S_{P R E S S}\right)$, and standard deviation of error of prediction $(S D E P)$. The $L M O$ crossvalidation was applied by deleting $25 \%$ of the compounds 
in four cycles and predicting the BBB permeability of compounds deleted in each cycle from the corresponding equations derived from the reduced data set. Some criteria for the reliability prediction and robustness of the models are suggested in Ref. [43-46]: $R^{2}>0.6$ and $Q_{L O(M) O}^{2}>0.5 ; R^{2} \geq$ $Q_{L O(M) O}^{2}$ and $Q_{L O O}^{2} \approx Q_{L M O}^{2}$

\subsubsection{Discriminant Function Analysis (DFA)}

Investigations of the CNS activity of the drugs analyzed throughout this study were based on the discriminant function analysis (DFA) using the physicochemical and chromatographic data connected with the BBB permeability and selected by MLR analysis. All results were compared with the models obtained and tested in the previous investigations [33,34]. In this DFA 111 structurally different compounds were assigned to the CNS+ or CNS- group of activity (defined according to Ref. [40], the remaining 43 compounds are not defined as $\mathrm{CNS}+$ /-). The classification functions were determined and validated for the compounds with the experimentally obtained BBB permeability (44 cases) and for compounds with calculated BBB permeability (B2) (111 cases).

Discriminant function analysis is a multivariate technique that has two purposes: to separate cases from distinct populations; and to allocate new cases into previously defined populations [47]. The DFA was performed by STATISTICA 10.0 [41] software. In all subsequently performed analyses the stepwise method was applied. The model was formed by introducing subsequent variables that mostly contributed to group discrimination. After introducing sufficient grouping variables to the model (i.e. after obtaining the maximum probability of a priori classification) discriminant functions (roots) discriminating the activity groups were calculated. The maximum number of functions will be equal to the number of groups minus one, or the number of variables in the analysis, whichever is less. The quality of the discriminant function was evaluated by Wilks' lambda parameter, which is a multivariate analysis of variance statistics that tests the quality of group means for the variable(s) in the discriminant function [47]. The Wilks' lambda can assume values in the range of 0 (perfect discrimination) to 1 (no discrimination) and the statistical significance of roots (discriminant functions) used for interpretation was established on the basis of $\chi^{2}$ tests of subsequent roots. Using statistically significant discriminant functions as the basis, canonical values were determined for the particular grouping variables. The scatter diagrams of the canonical values of the subsequent cases for the first two roots determined in the course of the analysis cannot be drawn to evaluate the discriminant power of the obtained models, because there are only two discriminated groups. The final phase of the qualitative analysis of the compounds was to determine the classification functions for each activity group. After calculation of the classification scores for a case, it is easy to decide how to classify it: in general we assign a case to a group for which it has the highest classification score. The tool used to determine how well the classification functions predict the group membership of cases is a classification matrix. The classification matrix shows the number of cases that were correctly classified (on the diagonal of the matrix) and those that were incorrectly classified.

The obtained discriminant models were evaluated by classification of 67 cases not included in the model (test set 47-113) with the known CNS+/- activity [40]. The values of the more important variables obtained with the DFA methodology were calculated for the test set. Than these values were introduced into the discriminant functions (12) and (13) obtained in validated DFA. We classify the case as belonging to the group for which it has the highest classification score. The new compounds were assigned to the CNS+ or CNS- group of activity.

Ethical approval: The conducted research is not related to either human or animal use.

\section{Results and discussion}

\subsection{Stepwise multiple regression analysis}

A number of computed or measured chromatographically (RP-18 TLC) BBB permeation descriptors have been presented in our previous papers [33,34,48]. The relationships between these descriptors and the observed (in vivo) BBB bioavailability were studied by $\mathrm{MR}$ and $\mathrm{DF}$ analysis for a group of compounds of diverse structures and successfully validated. The chromatographic data of the proposed analytical model (RP-18 TLC with the acetonitrile $-\mathrm{pH} 7.4$ phosphate buffered saline 70:30 (v/v) mobile phase) - $\mathbf{R}_{\mathrm{f}}$ and $\mathbf{R}_{\mathrm{f}} / \mathbf{P S A}$ were correlated with the parameters describing the ability of the compounds to penetrate the brain. The correlations are visible for the experimental BBB permeation as well as for the computed parameters B1, B2 and they are completed with the physicochemical parameters that are traditionally linked to the BBB permeation - $\log$ D, HD, HA, DM, $\log$ P, PSA, $\mathbf{S}_{\mathrm{a}}, \mathbf{M}_{\mathrm{w}}$ [49]. The other chromatographic data $\left(\mathbf{R}_{\mathrm{M}}, \mathbf{R}_{\mathrm{M}} / \mathbf{P S A}\right.$, 
$\mathbf{R}_{\mathrm{f}} / \mathbf{M}_{\mathrm{w}}, \mathbf{R}_{\mathrm{M}} / \mathbf{M}_{\mathrm{w}}$ ) were tested as the parameters describing the ability of the compounds to penetrate the brain, but only the $\mathbf{R}_{\mathrm{f}}$ and $\mathbf{R}_{\mathrm{f}} / \mathbf{P S A}$ descriptors are significant and have a predictive capability. The theoretical BBB permeation parameters are given by the already mentioned equations: B1 $(\log \mathrm{BB}=0.139+0.152 \log \mathrm{P}-0.0148 \mathrm{PSA})[31]$ and B2 $(\log \mathrm{BB}=0.547-0.016 \mathrm{PSA})$ [32]. It was demonstrated that the theoretical values $\mathbf{B 1}, \mathbf{B} 2$ describing the $\mathrm{BBB}$ permeability can be used to predict the BBB bioavailability of compounds even if they are not physically available [33]. A useful model of the blood-brain distribution was developed and validated. Models for discrimination between the CNS+ and CNS- compounds were built on the basis of the chromatographic and physicochemical parameters (HD, HA, $\mathbf{R}_{\mathrm{f}}, \mathbf{R}_{\mathrm{f}} / \mathbf{P S A}, \mathbf{D M}$ and $\mathbf{B 2}$ ) [33] and used successfully for a different group of compounds [34]. All compounds examined throughout these study were successfully assigned to one of two clusters: CNS+ or CNS-.

Such favorable results of analysis require confirmation. Our current research is aimed at proving the value of the previously proposed [33] analytical and mathematical models with the experiment involving a large (154 cases) and structurally diverse group of compounds.

The correlations of the BBB permeability factors (for the compounds with the experimentally obtained BBB permeability) with the chromatographic parameters $\left(\mathbf{R}_{\mathrm{f}}\right.$ and $\mathbf{R}_{\mathrm{f}} \mathbf{P S A}$ ) have been investigated (Equations (1)-(3)). The strongest relationship ( $\mathrm{R}=0.67, \mathrm{n}=46)$ can be found for B2 (Equation (3)), which is in line with the observation that simpler models are usually better [48]. All models contain the chromatographic parameters $\mathbf{R}_{\mathrm{f}}$ and $\mathbf{R}_{\mathrm{f}} /$ PSA.

$$
\begin{aligned}
& \text { B1 }=0.44( \pm 0.48)-1.91( \pm 0.72) \mathbf{R}_{\mathrm{f}}+16.39( \pm 7.21) \mathbf{R}_{\mathrm{f}} / \text { PSA } \\
& \mathrm{R}=0.44 ; \mathrm{R}^{2}=0.19 ; \mathrm{F}=5.1542 ; \mathrm{p}<0.00985 ; \mathrm{n}=46 ; \mathrm{s}=0.81335 \\
& \text { BB vivo }=0.48( \pm 0.47)+16.97( \pm 7.03) \mathbf{R}_{\mathrm{f}} / \mathbf{P S A}-1.15( \pm 0.69) \mathbf{R}_{\mathrm{f}}
\end{aligned}
$$

$\mathrm{R}=0.38 ; \mathrm{R}^{2}=0.15 ; \mathrm{F}=3.71 ; \mathrm{p}<0.03265 ; \mathrm{n}=46 ; \mathrm{s}=0.7824$ $\mathbf{B 2}=0.41( \pm 0.24)+16.63( \pm 4.75) \mathbf{R}_{\mathbf{f}} / \mathbf{P S A}-1.59( \pm 0.36) \mathbf{R}_{\mathbf{f}}(3)$

$R=0.67 ; R^{2}=0.44 ; F=17.050 ; p<0.0000 ; n=46 ; s=0.40861$

The experimental BBB permeability values for 46 compounds from the training set (1-46) were compared with the calculated $\mathbf{B} 1$ and $\mathbf{B} 2$ parameters and then with all the corresponding physicochemical parameters and chromatographic data. The resulting correlations differed significantly with $R=0.44$ for $\mathbf{B B}$ vivo/B1 and $R=0.55$ for BB vivo/B2, respectively; as B2 was in better agreement with the experimental values than B1, the former was used in further investigations.

At the next stage of these investigations the stepwise analysis was carried out using all the physicochemical parameters collected for 46 compounds from the training set. After the analysis the following relationship (4) was obtained, containing statistically significant descriptors:

$$
\begin{aligned}
& \text { BB vivo }=3.96( \pm 1.39)+0.14( \pm 0.048) \log \mathrm{D}+0.60( \pm 0.18) \\
& \text { B2 + 0.42( } \pm 0.15) \text { eH }-0.07( \pm 0.04) \mathbf{D M} \\
& \mathrm{R}=0.77 ; \mathrm{R}^{2}=0.59 \mathrm{~F}=14.594 ; \mathrm{p}<0.00000 ; \mathrm{s}=0.55729 ; \mathrm{n}=46
\end{aligned}
$$

On the basis of the stepwise analysis the parameter HA is introduced into the model. However, this parameter is too strongly correlated with another independent variable B2 so it had to be removed from the equation. One of the descriptors used in the model was again B2; additionally, the ability of the compound to cross the BBB depends on the distribution coefficient $(\log \mathbf{D})$, the total dipole moment (DM) and the energy of the highest occupied molecular orbital $(\mathbf{e H})$. The positive influence of $\log \mathbf{D}$ (the parameter that takes into account the molecule's ionization level) on the compound's partition between the phases is clearly visible. The model explains 59\% of the total variance.

The possibility of replacing the difficult-to-obtain $\mathbf{B B}$ vivo parameter with the $\mathbf{B} \mathbf{2}$ value as the $B B B$ penetration index was considered. Initially, the variables of the equation (4) given above were introduced, including HA and performing the regression analysis by the standard method:

$$
\begin{gathered}
\mathbf{B} 2=-0.28( \pm 0.58)-0.20( \pm 0.01) \mathbf{H A}-0.03( \pm 0.02) \mathbf{D M}+ \\
0.04( \pm 0.01) \log \mathbf{D}-0.09( \pm 0.06) \mathbf{e H} \\
\mathrm{R}=0.91 ; \mathrm{R}^{2}=0.83 ; \mathrm{F}(4,41)=49.484 ; \mathrm{p}<0.00000 ; \mathrm{s}= \\
0.23211 \mathrm{n}=46
\end{gathered}
$$

$$
\begin{gathered}
Q_{\text {LOO }}^{2}=0.77, S D E P=0.074572, P R E S S=3.43294, S_{P R E S S}= \\
0.273078, Q_{L M O}^{2}=0.71
\end{gathered}
$$

The result of the MR analysis for the $\mathbf{B} 2$ descriptor performed exclusively for the compounds with the known biological activity is very promising and reveals the importance of the same physicochemical parameters. The model explains $83 \%$ of the total variance. Next, the stepwise analysis by the forward selection method was performed. The result was very similar, although its statistical significance and the correlation coefficient $\mathbf{R}$ are higher. The resulting model explains $92 \%$ of the total variance in the studied group (46 compounds). 


$$
\begin{gathered}
\mathbf{B} 2=0.55( \pm 0.05)-0.14( \pm 0.01) \mathbf{H A}-0.03( \pm 0.02) \mathbf{D M}- \\
0.16( \pm 0.02) \mathbf{H D} \\
\mathrm{R}=0.96 ; \mathrm{R}^{2}=0.92 ; \mathrm{F}=163.84 ; \mathrm{p}<0.00000 ; \\
\mathrm{S}=0.15533 \mathrm{n}=46 \\
Q_{\text {LOO }}^{2}=0.90, S D E P=0.028056, P R E S S=1.290571 \\
S_{P R E S S}=0.167499, Q_{\text {LMO }}^{2}=0.88
\end{gathered}
$$

The $\mathbf{B} \mathbf{2}$ parameter may be successfully used as a measure of the BBB permeability. It is determined by the same molecular descriptors so it may replace the difficult-tomeasure BB in vivo parameter which makes it possible to expand the group of studied compounds by including the molecules without the BBB permeability determined in vivo (in this case to the total of 154 cases). The MR analysis performed with the physicochemical data confirms this conclusion. The equation (7) explains $88 \%$ of the total variance despite the threefold increase in the number of cases (n).

$$
\begin{gathered}
\text { B2 }=1.13( \pm 0,26)-0.14( \pm 0.01) \mathbf{H A}-0.19( \pm 0,01) \mathbf{H D}+ \\
0.02( \pm 0.00) \mathbf{e L}+0.07( \pm 0.02) \mathbf{e H} \\
\mathrm{R}=0.94 ; \mathrm{R}^{2}=0.88 ; \mathrm{F} 281.81 ; \mathrm{p}<0.0000 ; \mathrm{s}=0.23578 ; \\
\mathrm{n}=154 Q_{L O O}^{2}=0.87, S D E P=0.057613, P R E S S=9.448573, \\
S_{P R E S S}=0.240028, Q_{L M O}^{2}=0.86
\end{gathered}
$$

Our further investigations concentrated on the possibility of using reversed-phase thin layer chromatography to partially mimic the physiological conditions of crossing the BBB. It was assumed that the results of our biochromatographic experiments should improve the predictive capabilities of the purely computational models. The chromatographic data alone did not a give statistically significant correlation with BB vivo $(R=0.40)$. Molecular descriptors closely related to the compounds' bioavailability should, however, contribute to the predictive value of the model. The results of the stepwise MR analysis obtained after all the physicochemical parameters and chromatographic data had been introduced confirmed this assumption.

The analysis of the training set makes it possible to introduce to the computational model 8 independent variables (46 cases). The further stepwise analysis carried out with all the parameters, in 7 steps, results in the introduction of the additional variables, strongly connected with bioavailability. The chromatographic parameters $\mathbf{R}_{\mathbf{f}}$ and $\mathbf{R}_{\mathrm{f}} /$ PSA confirm the reasonable expectations connected with biochromatography. The descriptors equally often linked to the compound's ability to cross the

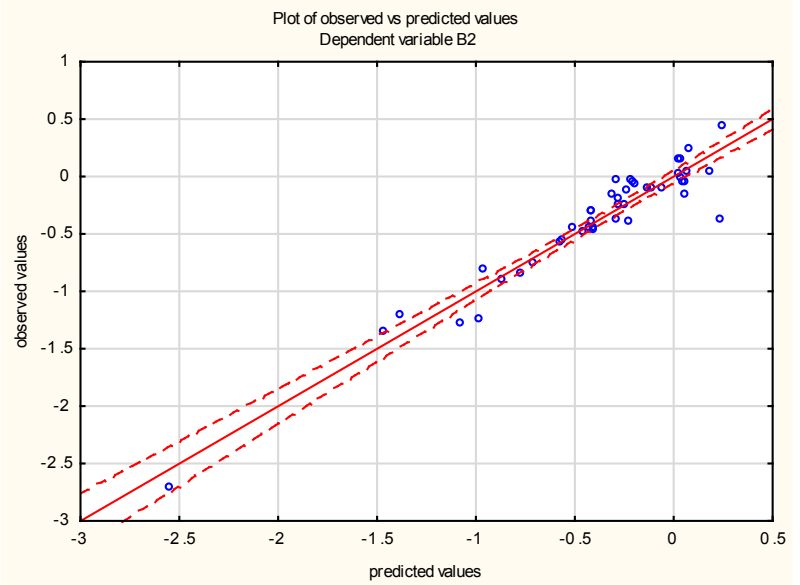

Figure 2: Plot of observed vs. predicted values. Dependent variable B2.

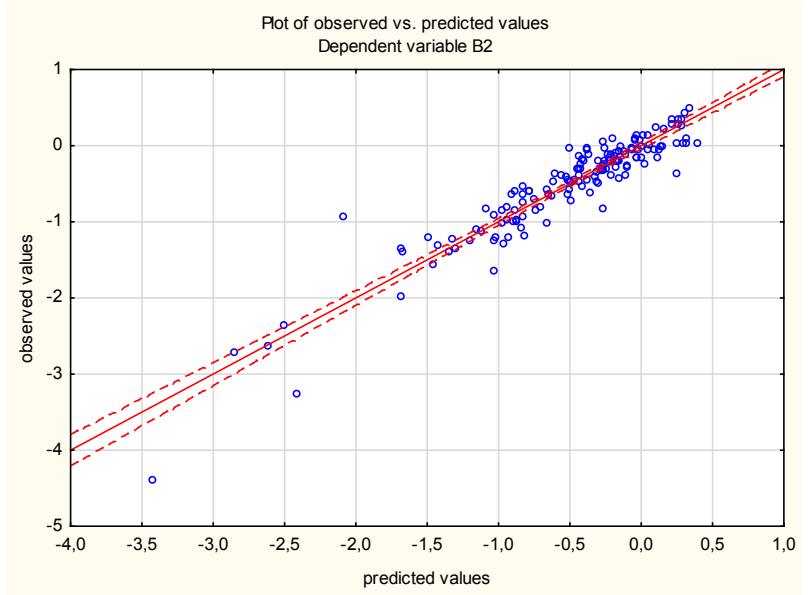

Figure 3: Plot of observed vs. predicted values (for model 7). Dependent variable $\mathbf{B} 2$.

BBB: $\log$ D, HA, eH, eL and V (combined here with the $\mathbf{R}_{\mathrm{f}}$ and $\mathbf{R}_{\mathbf{f}} / \mathbf{P S A}$ parameters) are present in the obtained model (8). The correlation coefficient is, however, not very high and the model explains $63 \%$ of the total variance.

$$
\begin{gathered}
\text { BB vivo }=1.78( \pm 1.60)+0.24( \pm 0.06) \log \mathbf{D}-0.17( \pm 0.06) \\
\mathbf{H A}+0.20( \pm 0.16) \mathbf{e H}+0.63( \pm 0.62) \mathbf{R}_{\mathrm{f}}-0.05( \pm 0.02) \mathbf{e L}+ \\
5.94( \pm 5.70) \mathbf{R}_{\mathrm{f}} / \mathbf{P S A}-0.11( \pm 0.11) \mathbf{V} \\
\mathrm{R}=0.80 ; \mathrm{R}^{2}=0.63 ; \mathrm{F} 8.9987 ; \mathrm{p}<0.00000 ; \\
\mathrm{S}=0.53719 ; \mathrm{n}=46
\end{gathered}
$$

The computed parameter $\mathbf{B} 2$ is well defined by the chromatographic data which confirms the applicability of chromatography to predict the CNS bioavailability. The model (3) explains $44 \%$ of the total variance. 
Table 2: Correlation matrix for model (9).

\begin{tabular}{|c|c|c|c|c|c|}
\hline & HD & HA & eH & $R_{f} / P S A$ & $R_{f}$ \\
\hline HD & 1.00000 & 0.58546 & -0.07994 & -0.26511 & 0.54010 \\
\hline HA & & 1.00000 & -0.08071 & -0.33889 & 0.32215 \\
\hline eH & & & 1.00000 & 0.06236 & 0.08418 \\
\hline$R_{f} /$ PSA & & & & 1.00000 & 0.17133 \\
\hline $\mathrm{R}_{\mathrm{f}}$ & & & & & 1.00000 \\
\hline
\end{tabular}

The relevance of the chromatographic parameters to the BBB permeability studies of 46 compounds of the known experimental BBB permeability (BB vivo) suggests the possibility of using these data to model the variance of B2. After the chromatographic data have been introduced, the regression model (9) is generated in 4 steps:

$$
\begin{gathered}
\mathbf{B} 2=-0.25( \pm 0.29)-0.15( \pm 0.01) \mathbf{H A}-0.15( \pm 0.01) \mathbf{H D}- \\
0.07( \pm 0.03) \mathbf{e H}+3.84( \pm 1.27) \mathbf{R}_{\mathrm{f}} / \mathbf{P S A} \\
\mathrm{R}=0.97 ; \mathrm{R}^{2}=0.94 ; \mathrm{F}=154.82 ; \mathrm{p}<0.00000 \mathrm{~s}=0.13519 ; \\
\mathrm{n}=46 Q_{\text {LOO }}^{2}=0.86, S D E P=0.040670, P R E S S=1.870843, \\
S_{P R E S S}=0.201669, Q_{\text {LMO }}^{2}=0.85
\end{gathered}
$$

This model explains the total variance in $94 \%$ and its predictive quality is validated. The influence of the $\mathbf{R}_{\mathbf{f}}$ data on the prediction of the $\mathbf{B} \mathbf{2}$ variance has been studied as well. The regression model containing the variables: $\mathbf{H A}, \mathbf{H D}, \mathbf{e H}$ and $\mathbf{R}_{\mathrm{f}}$ explains $92 \%$ and the model based on $\mathbf{H A}, \mathbf{H D}, \mathbf{e H}, \mathbf{R}_{\mathrm{f}} / \mathbf{P S A}$ and $\mathbf{R}_{\mathrm{f}} 93 \%$ of the total variance, respectively. The independent variables are not intercorrelated. This confirms the predictive capability of both chromatographic descriptors and the selection of the equation (9) as the $\mathbf{B} 2$ variability model.

The correlation between $\mathbf{B} \mathbf{2}$ and the chromatographic data generated on the basis of all 154 cases (including the test set 1-46) gives an equation that explains $31 \%$ of B2 variance $(R=0.56 ; n=154)$. The molecular descriptors and the chromatographic data as described above were used to generate the model given by Equation (10) for the whole group of 154 compounds including the test set. The outcome of this analysis is very good - the model explains over $90 \%$ of the total variance.

$$
\begin{gathered}
\mathbf{B} 2=0.93( \pm 0.27)-0.15( \pm 0.01) \mathbf{H A}-0.19( \pm 0.27) \mathbf{H D} \\
+0.06( \pm 0.02) \mathbf{e H}+0.02( \pm 0.00) \mathbf{e L}+0.16( \pm 0.10) \mathbf{R}_{\mathrm{f}}+ \\
1.78( \pm 1.09) \mathbf{R}_{\mathrm{f}} / \mathbf{P S A}
\end{gathered}
$$

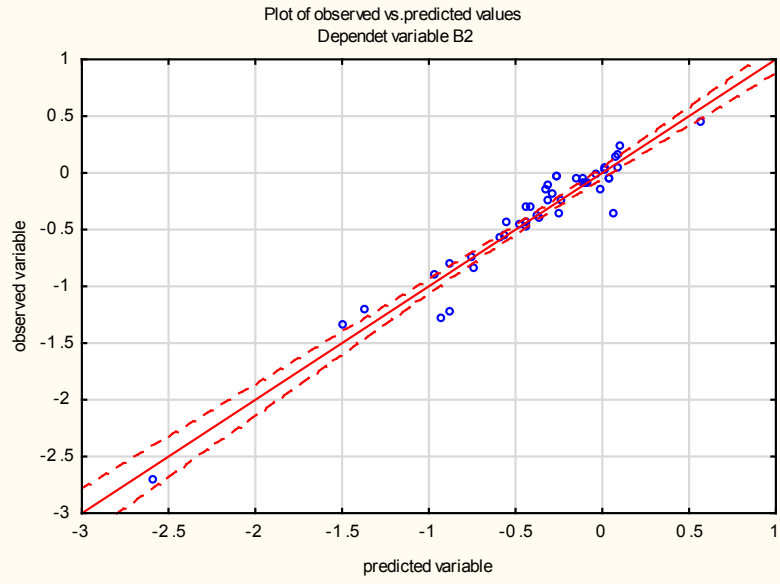

Figure 4: Plot of observed vs. predicted values for model (9). Dependent variable B2.

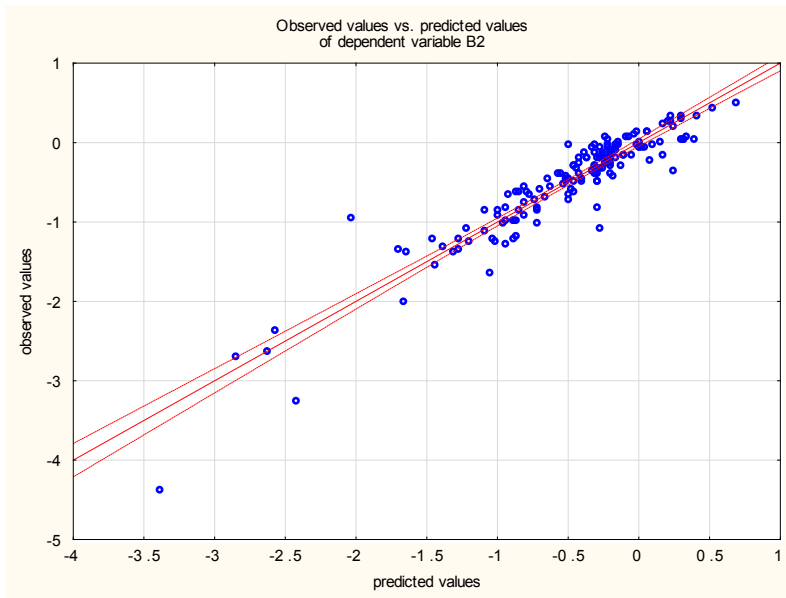

Figure 5: Plot of observed vs. predicted values for model (10). Dependent variable B2.

$\mathrm{R}=0.94 ; \mathrm{R}^{2}=0.90 ; \mathrm{F}=196.52 ; \mathrm{p}<0.00000 \mathrm{~s}=0.23038 ; \mathrm{n}=154$

$Q_{\text {LOO }}^{2}=0.87, S D E P=0.059787$, PRESS $=9.207211, S_{\text {PRESS }}=$ $0.244514, Q_{\text {LMO }}^{2}=0.86$. 
Table 3: Correlation matrix for models (10) and (11).

\begin{tabular}{|c|c|c|c|c|c|c|}
\hline & Rf & Rf/PSA & HD & HA & $\mathrm{eH}$ & $\overline{\mathrm{el}}$ \\
\hline $\mathrm{Rf}$ & 1.00000 & 0.17063 & 0.42218 & 0.37710 & 0.06021 & -0.09090 \\
\hline Rf/PSA & & 1.00000 & -0.21772 & -0.31267 & 0.13479 & 0.20615 \\
\hline HD & & & 1.00000 & 0.55937 & -0.02446 & -0.30065 \\
\hline HA & & & & 1.00000 & -0.13247 & $-0,44623$ \\
\hline $\mathrm{eH}$ & & & & & 1.00000 & 0.03204 \\
\hline $\mathrm{eL}$ & & & & & & 1.00000 \\
\hline
\end{tabular}

The MR analysis based on the independent variables given by the $\mathbf{B} \mathbf{2}$ variability model presented earlier [33] has been repeated. The model obtained in this manner explained 95\% of the variance for the group of 34 cases and involved the variables $\mathbf{H A}, \mathbf{H D}, \mathbf{R}_{\mathrm{f}} / \mathbf{P S A}$ [33]. The resulting equation (11) has very good parameters and confirms the value of this model describing the properties of any group of cases. The model is resistant to a significant increase in the number of cases.

$$
\begin{gathered}
\mathbf{B} 2=0.46( \pm 0.05)-0.16( \pm 0.01) \mathbf{H A}-0.19( \pm 0.01) \mathbf{H D}+ \\
2.94( \pm 1.09) \mathbf{R}_{\mathrm{f}} / \mathbf{P S A}
\end{gathered}
$$

$\mathrm{R}=0.93 ; \mathrm{R}^{2}=0.90 ; \mathrm{F}=342.74 ; \mathrm{p}<0.00000 \mathrm{~s}=0.24540 ; \mathrm{n}=154$ $Q_{\text {LOO }}^{2}=0.85, S D E P=0.068583, P R E S S=10.561839, S_{\text {PRESS }}=$ $0.261884, Q_{L M O}^{2}=0.85$.

There are many parameters that enable the simplified prediction of the ability of drugs to enter the CNS. The molecular descriptors, describing the physicochemical properties of solutes and gathered in the "Rule of 5" have been selected after the analysis of thousands of compounds.

Complex patterns of drug - BBB interactions in vivo lead to discrepancies of the results of this rule application. Numerous sorptions and desorptions of a drug over its distribution path intensify these differences. Studying the structure and physicochemical properties arising from the compound structure is crucial but usually insufficient for the quantitative description of the distribution effect.

All the parameters proposed in the "Rule of 5” have the limited ranges of effectiveness for the BBB permeation (CNS+). These ranges are usually quite narrow and their extension or shifting is usually connected with other properties of a compound. There are very few mathematical models of biological activity based on a single independent value. Hence, the prediction of a drug brain absorption exclusively on the basis of its

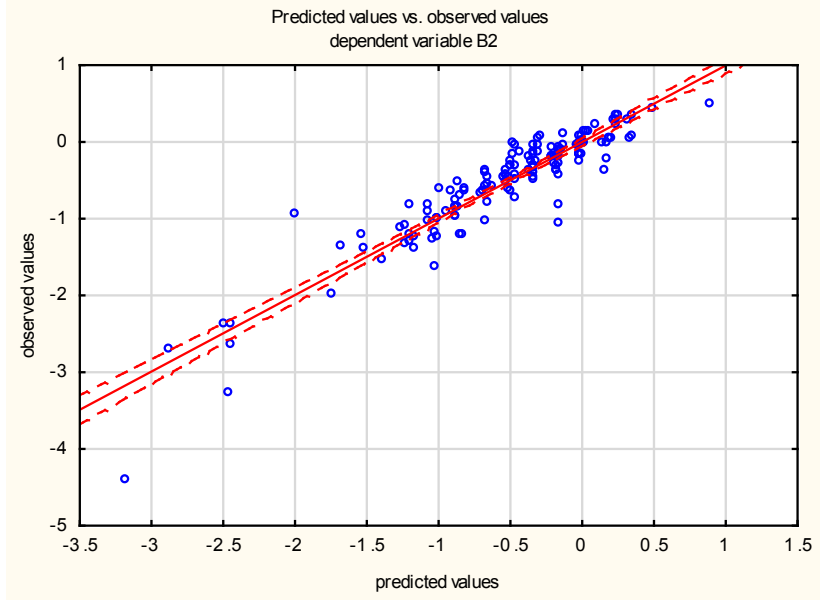

Figure 6: Predicted vs. observed values for model (11). Dependent variable B2.

physicochemical properties (e.g. "Rule of 5") has certain limitations. Descriptors derived from these parameters are used. Linking two single parameters has given rise to the descriptors such as: $\log \mathbf{P}-\mathbf{N}+\mathbf{0}, \mathbf{\% P S A}$ (PSA and $\mathbf{S}_{A}$ ). Frequently used logarithmic derivatives (e.g. $\log \mathbf{M}_{\mathrm{w}}$ ) facilitate a better fit to the complex behavior phenomena (with the tendency to reach a state of equilibrium) or to other logarithmic measures of activity $\left(\mathbf{p A}_{2}, \mathbf{p D _ { 2 }}, \log \mathbf{B B}\right.$ etc.).

If compounds under investigation are available, their activity may be studied in specially designed analytical models, mimicking the conditions in vivo. The subtle differences in behavior must be multiplied to become traceable and expressed quantitatively. The application of a chromatographic method gives such possibilities both in studying the CNS bioavailability $[29,33,34]$ and in other areas of medicinal chemistry [50]. The analytical models are used to study and compare the solutes of a great diversity of physicochemical properties. In the case of the chromatographic models the phase systems 
are required that would make it possible to study all interesting cases without changing the conditions. Every chromatographic model imitates the complex conditions of in vivo interactions and must, in a simplified way, represent a specific phenomenon, not the universal features of the basic chromatographic separation. This is the reason why it is essential to introduce special features related to the studied biological activity. The phase affinity in the adsorption retention mechanism is of a hydro-lipophilic nature and the hydrophobic interactions are also important. The RP-18 phase exhibits the features of a pseudo-adsorptive support.

Introducing a measure of the analyte polar surface area refines the effect of the chromatographic separation $\left(\mathbf{R}_{\mathrm{f}}\right)$ with the properties strongly connected with the modeled phenomenon of the BBB permeation. It seems that taking into account the polarity of the molecules surface extends the possibility of studying the solutes of different structures. The variation of $\mathbf{R}_{\mathrm{f}} / \mathbf{P S A}$ is larger than that of $\mathbf{R}_{\mathrm{f}}$ for the studied group of compounds.

In the experiment for 111 cases presented above (21 cases with the defined CNS- parameter and 90 cases from the $\mathbf{C N S}+$ group), the values of the $\mathbf{R}_{\mathrm{f}} / \mathbf{P S A}$ parameter were $\geq 0.009$ for CNS+ (14 errors with values 0.004-0.008) and $<0.009$ for CNS- (3 errors with values 0.0092-0.0099); the values of $\mathbf{R}_{\mathbf{f}}$ for the same group were 0.10-0.90 for CNS+ and 0.50-0.90 for CNS-. These results clearly indicate the greater stability of the $\mathbf{R}_{\mathrm{f}} / \mathbf{P S A}$ parameter towards the predicted $\mathrm{BBB}$ permeability.

The parameter B2 is also very stable in this experiment. In the group of 111 cases with the defined CNS+/- bioavailability the values of $\mathbf{B} 2$ for 90 CNS+ cases were $\geq-0.9$ ( 6 errors were detected with the values -0.97 to -1.38$)$. The values of the $\mathbf{B} 2$ parameter in the group of 21 CNS- cases were $<-0.9$ ( 3 errors were detected with the values 0.41 to -0.81 ). The analytes with the known permeability descriptor BBB vivo were within the same limits. Out of 6 CNS- cases 2 solutes were misclassified and in the group of 39 CNS + compounds the number of incorrectly classified compounds was also 2 .

The values of the BBB vivo parameter were $>-0.9$ for CNS+ ( 3 errors in the group of 30 cases) and $<-0.9$ for CNS- (6 cases without errors).

\subsection{Discriminant Function Analysis}

Drugs whose ability to cross the BBB has been measured experimentally are often described in the literature and databases [40,48] as CNS positive (CNS+) or CNS negative (CNS-). The qualitative parameter CNS+/CNS- may be used
Table 4: Classification matrix for model with discriminating variables (B2, eL and $\left.R_{f} / P S A\right)(12,13)$.

\begin{tabular}{llll}
\hline observed & $\begin{array}{l}\text { Percentage of cases correctly } \\
\text { classified }\end{array}$ & CNS- & CNS+ \\
\hline CNS- & 83.3333 & 5 & 1 \\
CNS+ & 100.0000 & 0 & 38 \\
Total & 97.7273 & 5 & 39 \\
\hline
\end{tabular}

as a grouping variable in DFA to establish classification functions to predict the bioavailability of new drugs. 111 compounds from the training set of 154 cases were found in the database [40] and separated into two groups: CNS(21 cases, code 0 ) and $\mathbf{C N S}+$ (90 cases, code 1 ).

The stepwise DFA was carried out for 44 cases: $\underline{\mathbf{1}} \underline{\mathbf{9}}$, $\underline{11}-\underline{31}$ and $\underline{33}-\underline{46}$ with the measured BBB permeability BB vivo (for two solutes - acyclovir and tibolon no $\mathrm{CNS}+$ /classification has been found in Ref. [40]) on the basis of standardized data, using the chromatographic and physicochemical parameters selected in the course of the multiple regression analysis (8): $\log \mathbf{D}, \mathbf{H A}, \mathbf{e H}, \mathbf{R}_{\mathrm{f}}, \mathbf{B} 2, \mathbf{e L}$, $\mathbf{R}_{\mathrm{f}} / \mathbf{P S A}$. Almost full separation of two groups: CNS- and CNS+ was achieved in three steps, with B2, eL and $\mathbf{R}_{\mathrm{f}} / \mathbf{P S A}$ as discriminating variables, which additionally confirms the value of the chromatographic parameter $\mathbf{R}_{\mathrm{f}} / \mathbf{P S A}$ in describing the compounds' ability to cross the BBB. The subsequent results in the form of classification matrices and functions are presented below.

The classification functions for each group of activity CNS- and CNS+ were calculated:

$$
\begin{gathered}
\text { CNS- }=-6.47816-5.58576 \mathbf{B} 2+1.24573 \mathbf{e L}+ \\
0.80487 \mathbf{R}_{\mathbf{f}} / \mathbf{P S A} \\
\text { CNS }+=-0.292523+1.020848 \mathbf{B} 2- \\
0.322966 \mathbf{e L}-0.106046 \mathbf{R}_{\mathbf{f}} / \mathbf{P S A}
\end{gathered}
$$

The outcome of the lastDFA was verified demonstrating the high classification power of the model (Wilks' lambda parameter $=0.394087, \chi^{2}=37.71291$, p-level $=0.000000$ ).

The reliability of the model derived from DFA was determined by a cross-validation test based on the leaveone-out methodology. 44 cases $(\underline{1}-\underline{9}, \underline{11}-\underline{31}, \underline{33}-\underline{46})$ with the measured BBB permeability (BB vivo) were examined. The procedure is described above. The results obtained with the methodology are summarized in a cross-validation matrix (Table 5). Using the results obtained via the cross-validation methodology we can confirm the reliability of the DFA model (the cross-validation error is $0 \%$ ). The examination of the cross-validation matrix suggested that the classification 
probability was the same as the classification probability obtained a posteriori (97.73\%) (Table 5). The reliability of the analyses was proved. The models presented above not only described precisely the investigated groups of cases but have diagnostics value for new cases.

In order to validate the methods and confirm the discriminating value of the descriptors selected in the course of this study a group of 67 cases without the measured BBB vivo but with the BBB permeability known from the database [40] (47-113) was introduced. The predictive values of the determined classification functions (equations: (12) and (13)) proposed for the classification of compounds as CNS+/- were assessed. For this purpose, the variables calculated for the other compounds ( $\underline{47}-\underline{113})$ with the known BBB permeability: CNS- (15 cases) and CNS+ (52 cases) were added to the raw variable file for the compounds $\underline{1-9}, \underline{11}-\underline{31}, \underline{33}-\underline{46}$ and then standardized together.

The classification functions: (12) and (13) were subsequently applied to calculate the appropriate qualification values for the compounds 47-52, 62-70 - 15 cases CNS- and 53-61, 11-113 - 52 cases CNS+ (Table 6, Supplementary Materials).

The effectiveness of the classification functions calculated in the previous paper (Equations 9 and 10 in Ref. [33]) and used successfully in Ref. [34] has been investigated for the cases $\mathbf{4 7 - 1 1 3}$. The results are presented in Table 6.

$$
\begin{aligned}
\text { CNS }-= & -10.8254 \text { B2 }+3.2154 \mathbf{R}_{\mathrm{f}} / \text { PSA }-6.3785 \\
& \quad \text { Equation }(\mathbf{9}) \text { in Ref. [33]) } \\
\text { CNS }+= & 5.32916 \text { B2 }-1.76240 \mathbf{R}_{\mathbf{f}} / \text { PSA }-1.00757 \\
& \text { (Equation (10) in Ref. [33]) }
\end{aligned}
$$

On the basis of the Equation (12) 6 out of 15 CNS- cases were misclassified vs. 2 out of $52 \mathrm{CNS}+$ compounds misclassified according to the Equation (13). The total number of correctly classified cases reached $88.06 \%$.

At the same time, the application of the classification functions established in the previous DFA (Equations 9 and 10 in Ref. [33]) and presented below furnished 1 (out of 15) misclassified CNS- case and 7 (out of 52) misclassified CNS + cases. The total number of correctly classified cases reached $88.06 \%$.

The investigation of cases with the known BB vivo parameter revealed 6 CNS- compounds (2 classification errors) and 38 CNS + cases with 5 classification errors, respectively. The results confirm the value of the chromatographic parameters $\mathbf{R}_{\mathrm{f}} / \mathbf{P S A}$ and $\mathbf{B} \mathbf{2}$ in simple defining the ability of compounds to cross the BBB. The total number of the correctly classified cases was $84.09 \%$.
Table 5: Cross-validation matrix.

\begin{tabular}{ll}
\hline observed & Percentage of cases correctly classified \\
\hline CNS- & 83.3333 \\
CNS+ & 100.0000 \\
Total & 97.7273 \\
\hline
\end{tabular}

Table 7: Classification matrix for model with discriminating variables (B2, eL, HA, $\log D$ and $\left.R_{f} / P S A\right)(14,15)$.

\begin{tabular}{llll}
\hline observed & $\begin{array}{l}\text { Percentage of cases correctly } \\
\text { classified }\end{array}$ & CNS- & CNS+ \\
\hline CNS- & 66.66666 & 14 & 7 \\
CNS+ & 97.77778 & 2 & 88 \\
Total & 91.89189 & 16 & 95 \\
\hline
\end{tabular}

Table 8: Cross-validation matrix.

\begin{tabular}{ll}
\hline observed & Percentage of cases correctly classified \\
\hline CNS- & 67.83 \\
CNS+ & 97.74 \\
Total & 92.04 \\
\hline
\end{tabular}

Just like in the case of the training set $(\mathbf{1}-\underline{9}, \underline{11}-\underline{31}$, 33-46) the stepwise DFA was carried out for the set of all compounds with defined CNS+/- (111 cases: 1-9, $\underline{\mathbf{1 1}}-\underline{\mathbf{3 1}}$ and 33-113) involving the independent variables used in the previous analyses (Equations (14) and (15)). The classification matrix for the total of 111 cases is given in Table 7.

$$
\begin{gathered}
\text { CNS }-=-3.72522+0.68546 \mathbf{H A}+0.54721 \mathbf{e L}-0.64253 \mathbf{~ l o g} \\
\mathbf{D}-2.31095 \mathbf{B} \mathbf{2}+0.45783 \mathbf{R}_{\mathbf{f}} / \mathbf{P S A}
\end{gathered}
$$

$$
\begin{gathered}
\text { CNS }+=-0.281386+0.008041 \mathbf{H A}-0.5091631 \mathbf{e L}+ \\
0.040695 \log \mathbf{D}+0.504695 \mathbf{B} 2+0.46771 \mathbf{R}_{\mathrm{f}} / \mathbf{P S A} \text { (15) }
\end{gathered}
$$

The quality of DFA leading to the model (14)/(15) was verified by Wilks' lambda parameter 0.531980; $\chi^{2}$ $=67.21731 ; \mathrm{p}$-level $=0.000000$ confirmed the model's high discriminating potential. Only 9 cases in the group of 111 compounds were incorrectly classified. Examination of the cross-validation matrix proved that the classification probability was higher than the classification probability obtained a posteriori (Table 8). 


\section{Conclusions}

The results of our studies confirmed the values of the previously proposed [33] analytical and mathematical models for the experiment with a large and structurally diverse group of compounds. The presented results show that the proposed analytical and mathematical models are universal and can be applied to any chemical compound.

The chromatographic data $\left(\mathbf{R}_{\mathrm{f}}\right.$ and $\left.\mathbf{R}_{\mathrm{f}} / \mathbf{P S A}\right)$ of the proposed analytical model (RP-18 TLC) are correlated with the parameters describing the ability of the compounds to penetrate the brain. The chromatographic parameters $\left(\mathbf{R}_{\mathrm{f}}\right.$ and especially $\mathbf{R}_{\mathrm{f}} / \mathbf{P S A}$ ) obtained from the previous studies have a universal predictive capacity and can be therefore considered as one of the descriptors supplementing the "Rule of 5" parameter group [49].

The correlations are visible for the computed parameter B2 and they are completed with the physicochemical parameters that are traditionally linked to the BBB permeation - $\log$ D, HD, HA, DM (,Rule of 5", Ref. [49]). The results of the simple, inexpensive and very rapid chromatographic analysis proposed in this study may be applied to predict the BBB permeability of compounds isolated or synthetized on a very small scale.

The parameter B2, given by the equation $\mathbf{B} 2=\log \mathrm{BB}=$ $0.547-0.016$ PSA [32] has a predictive and discriminating value corroborated by the equations (12)-(15). The confirmed value of the computed BBB bioavailability indicator can be used to predict the BBB bioavailability of compounds without the experimental BBB permeability data [48]. The proposed chromatographic model of the BBB permeation based on RP-18 TLC and the computed B2 parameter can be used in future investigations of compounds of different chemical structures. The results of this and earlier $[33,34]$ investigations confirm not only the assumed ability of compounds to cross the BBB but also the universal character and efficiency of all generated mathematical and analytical models to predict the BBB permeability of compounds available on a very small scale (sufficient for thin-layer chromatographic analysis) or even physically unavailable.

Taking into consideration the great importance of the calculated log BB descriptors - B1 and $\mathbf{B} 2$ revealed in the earlier analyses [33,34,48], a new element theoretically connected with the BBB permeation has been introduced that describes the molecular structure and is present in the B1 and B2 algorithms [31,32] (PSA value). A new descriptor $\mathbf{R}_{\mathrm{f}} / \mathbf{P S A}$ has been created.

Introducing a measure of the analyte polar surface area refines the effect the chromatographic separation $\left(\mathbf{R}_{\mathrm{f}}\right)$ with the properties strongly connected with the modeled phenomenon of the BBB permeation. It seems that taking into account the polarity of the molecules surface extends the possibility of studying the solutes of different structures. The variation of $\mathbf{R}_{\mathrm{f}} / \mathbf{P S A}$ is larger than that of $\mathbf{R}_{\mathbf{f}}$ for the studied group of compounds. All the experiments performed so far [33,34] confirm the importance of the $\mathbf{R}_{\mathrm{f}} /$ PSA parameter as the value closely related to the CNS+/descriptor.

It was therefore concluded that the reversed-phase thin layer chromatographic data obtained on the RP-18 $\mathrm{F}_{254 \mathrm{~s}}$ support and with acetonitrile-pH 7.4 phosphate buffered saline 70:30 (v/v) mobile phase may be used as the BBB permeability descriptors. The range of the $\mathbf{R}_{\mathrm{f}} /$ PSA parameter indicating the likely CNS bioavailability was, for the group of 34 cases studied earlier [33], $\geq 0.009$ for CNS+ and $<0.009$ for CNS- (without the errors). In an identical experiment for 111 cases presented above, the values of the $\mathbf{R}_{\mathrm{f}} / \mathbf{P S A}$ parameter were $\geq 0.009$ for $\mathbf{C N S}+$ and $<0.009$ for CNS-. These results clearly indicate the greater stability of the $\mathbf{R}_{\mathrm{f}} / \mathbf{P S A}$ parameter towards the predicted BBB permeability.

The parameter $\mathbf{B} \mathbf{2}$ is also very stable in this experiment. In the group of 111 cases with the defined CNS+/- bioavailability the values of $\mathbf{B 2}$ for 90 CNS+ cases were $\geq-0.9$. The values of the $\mathbf{B} 2$ parameter in the group of 21 CNS- cases were $<-0.9$.

The values of the BBB vivo parameter were $>-0.9$ for CNS+ and $<-0.9$ for CNS-.

Acknowledgements: This research was supported by an internal grant of the Medical University of Łódź no. 503/3-016-03/503-31-001. The authors are very grateful to the companies: CIBA, Polfa Pabianice, Symrise, BASF and Merck for the samples of compounds used throughout this study.

Conflict of interest: Authors declare no conflict of interest.

\section{References}

[1] Abbott N.J, Patabendige A.A.K., Dolman D.E.M., Yusof S.R., Begley D.J., Structure and function of the blood-brain barrier, Neurobiol. Dis. 2010, 37, 13-25.

[2] Abbot N.J., Blood-brain barrier structure and function and the challenges for CNS drug delivery, J. Inherit. Metab. Dis. 2013, 36, 437-449.

[3] Misra A., Ganesh S., Shahiwala A., Shah S.P., Drug delivery to the central nervous system: a review, J. Pharm. Pharm. Sci. 2003, 6, 252-73. 
[4] Pardridge W.M., Log(BB), PS products and in silico models of drug brain penetration, Drug Disc. Today 2004, 9, 392-393.

[5] Shityakov E., Salvador E., Foster C., In silico, in vitro and in vivo methods to analyse drug permeation across the blood-brain barrier: A critical review, OA Anaestethics 2013, 1, http://www. oapublishinglondon.com/article/913\#

[6] Abbot N.J., Prediction of blood-brain permeation in drug discovery from in vivo, in vitro and in silico models, Drug Disc. Today: Technol. 2004, 1, 407-416.

[7] Wilhelm I., Fazakas C., Krizbai I.A., In vitro models of the bloodbrain barrier, Acta Neurobiol. Exp. (Wars.) 2011, 71, 113-128.

[8] Reis J. M., Sinko B.C., Serra H.R., Parallel Artificial Membrane Permeability Assay (PAMPA) - Is it Better than Caco-2 for Human Passive Permeability Prediction? Mini Rev. Med. Chem. 2010, 10, 1071-1076.

[9] Meyer H., Zur Theorie der Alkoholnarkose, Arch. Exp. Path. Pharm. 1899, 42, 109-118.

[10] Hansch C., Steward A.R., Anderson S.M., Bentley D.L., Parabolic dependence of drug action upon lipophilic character as revealed by a study of hypnotics, J. Med. Chem. 1967, 11, 1-11.

[11] Timmermans P.B.W.M., Brands A., van Zwieten P.A., Lipophilicity and Brain Disposition of Clonidine and Structurally Related Imidazolidines, Naunyn-Schmiedeberg's Arch. Pharmacol. 1977, 300, 217-226.

[12] Dischino D.D., Welch M.J., Kilbourn M.R., Raichle M.E., Relationship Between Lipophilicity and Brain Extraction of C-11Labeled Radiopharmaceuticals, J. Nucl. Med. 1983, 24, 10301038.

[13] Young R.C., Mitchell R.C., Brown T.H., Ganellin C.R., Griffiths R., Jones M., et al., Development of a new physicochemical model for brain penetration and its application to the design of centrally acting $\mathrm{H} 2$ receptor histamine antagonists, J. Med. Chem. 1988, 31, 656-671.

[14] Ciura K., Dziomba S., Nowakowska J., Markuszewski M.J., Thin layer chromatography in drug discovery process, J. Chromatogr. A 2017, 1520, 9-22.

[15] Berthod A., Carda-Broch S., Determination of liquid-liquid partition coefficients by separation methods, J. Chromatogr. A 2004, 1037, 3-14.

[16] Poole S.K., Poole C.F., Separation methods for estimating octanol-water partition coefficients, J. Chromatogr. B 2003, 797, 3-19.

[17] Bate-Smith E.C., Westall R.G., Chromatographic behaviour and chemical structure I. Some naturally occurring phenolic substances, Biochem. Biophys. Acta 1950, 4, 427-440.

[18] Soczewiński E., Wachtmeister C.A., The relation between the composition of certain ternary two-phase solvent systems and $R_{m}$ values, J. Chromatogr. 7 (1962) 311-320.

[19] Flieger J., Tatarczak M., Effect of Inorganic Salts as Mobile-Phase Additives on Lipophilicity Values Determined by Reversed-Phase Thin-Layer Chromatography for New 1,2,4-Triazole Derivatives, J. Planar Chromatogr. 2006, 19, 386-392.

[20] Onisor C., Posa M., Kevresan S., Kuhajda K., Sarbu C., Estimation of chromatographic lipophilicity of bile acids and their derivatives by reversed-phase thin layer chromatography. J. Sep. Sci. 2010, 33, 3110-3118.

[21] Djakovic-Sekuli T., Perisic-Janjic N., Djurendi E., Retention data from reverse-phase high-performance thin-layer chromatography in characterization of some bis-salicylic acid derivatives, Biomed. Chromatogr. 2009, 23, 881-887.
[22] Vastag D., Perisic-Janjic N., Tomic J., Petrovic S., Evaluation of the Lipophilicity and Prediction of Biological Activity of Some N-Cyclohexyl-N-Substituted-2- Phenylacetamide Derivatives Using RP-TLC, J. Planar Chromatogr. 2011, 24, 435-440.

[23] Perisic-Janjic N., Kaliszan R., Milosevic N., Uscumlic G., Banjac N., Chromatographic retention parameters in correlation analysis with in silico biological descriptors of a novel series of $\mathrm{N}$-phenyl-3-methyl succinimide derivatives, J. Pharm. Biomed. Anal. 2013, 72, 69-73.

[24] Milosevic N.P., Stojanovic S.Z., Penov-Gasi K., Perisic-Janjic N., Kaliszan R., Revised- and normal-phase liquid chromatography in quantitative structure retention-property relationship of newly synthesized seco-androstene derivatives, J. Pharm. Biomed. Anal. 2014, 88, 636-642.

[25] Apostolov S., Vastag D., Matijevic B., Nakomcic J., Marinkovic A., Studying retention behavior, lipophilicity and pharmacokinetic characteristics of $\mathrm{N}$-substituted phenyl-2-chloroacetamides, Contemp. Mater. 2014, V-1, 101-110.

[26] Jevric L.R., Podunavac-Kuzmanovic S.O., Svarc-Gajic J.V., Kovacevic S.Z., Jovanovic B.Z., RP-HPTLC Retention Data in Correlation with the In-silico ADME Properties of a Series of s-Triazine Derivatives, Iran. J. Pharm. Res. 2014, 13, 1203-1211.

[27] Kovacevic S.Z., Jevric L.R., Podunavac-Kuzmanovic S.O., Loncar E.S., Prediction of In-silico ADME Properties of 1,2-0-Isopropylidene Aldohexose Derivatives. Iran. J. Pharm. Res. 2014, 13, 899-907.

[28] Komsta Ł., Skibiński R., Bojarczuk A., Radoń M., Salting-Out Chromatography - A Practical Review, Acta Chromatogr. 2011, 23, 191-203.

[29] Ciura K., Belka M., Kawczak P., Bączek T., Markuszewski M.J., Nowakowska J., Combined computational-experimental approach to predict blood-brain barrier (BBB) permeation based on "green" salting-out thin layer chromatography supported by simple molecular descriptors, J. Pharm. Biomed. Anal. 2017, 143, 214-221.

[30] Pajouhesh H., Lenz G.R., Medicinal Chemical Properties of Successful Central Nervous System Drugs, NeuroRx 2005, 2, 541-553.

[31] Norinder U., Haeberlein M., Computational approaches to the prediction of the blood-brain distribution, Adv. Drug Deliv. Rev. 2002, 54, 291-313.

[32] Clark D.E., Rapid Calculation of Polar Molecular Surface Area and Its Application to the Prediction of Transport Phenomena. 2. Prediction of Blood-Brain Barrier Penetration, J. Pharm Sci. 1999, 88, 815-821.

[33] Sobańska A.W., Brzezińska E., Application of RP-18 Thin Layer Chromatography and Quantitative Structure-Activity Relationship Analysis for the Prediction of the Blood-Brain Barrier Permeation, J. Planar Chromatogr. 2016, 29, 287-298.

[34] Pyzowski J., Brzezińska E., Sobańska A.W., RP-18 Chromatographic-Based Study of the Blood-Brain Barrier Permeability od Selected Sunscreens and Preservatives, J. Planar Chromatogr. 2017, 30, 275-284.

[35] Komsta Ł., Skibiński R., Berecka A., Gumieniczek A., Radkiewicz B., Radoń M., Revisiting thin-layer chromatography as a lipophilicity determination tool-A comparative study on several techniques with a model solute set, J. Pharm. Biomed. Anal. 2010, 53, 911-918.

[36] Sobańska A.W., Wójcicka K., Brzezińska E., Evaluation of the lipophilicity of selected sunscreens-A chemometric analysis of 
thin-layer chromatographic retention data, J. Sep. Sci. 2014, 37, 3074-3081.

[37] HyperChem for Windows Release 7.02, HyperCube Inc, Gainesville, FL, USA, 2002.

[38] Stewart J.J.P., MOPAC: a semiempirical molecular orbital program, J. Comput. Aided Mol. Des. 1990, 4, 1-105.

[39] ACD/Labs ${ }^{\mathrm{TM}}$ Log D Suite 8.0, pKa dB 7.0, Advanced Chemistry Development Inc., Toronto, Canada, 2004.

[40] Drugbank: https://www.drugbank.ca/, Edmonton, Canada, 2018

[41] Vilar S., Chakrabarti M., Constanzi S., Prediction of passive blood-brain partitioning: straightforward and effective classification models based on in silico derived physicochemical descriptors, J. Mol. Graph. Model. 2010, 28, 899-903.

[42] STATISTICA V. 10.0 StatSoft, Tulsa, OK, USA, 2011

[43] Tropsha A., Gramatica P., Gombar V.K., The importance of being earnest: validation is the absolute essential for successful application and interpretation of QSPR models, QSAR Comb. Sci. 2003, 22, 69-77.

[44] Tropsha A., Best practices for QSAR model development, validation and exploitation, Mol. Inf. 2010, 29, 476-488.

[45] Wold S., Validation of QSAR, Quant. Struct. Act. Relat. 1991, 10, 191-193.

[46] Kiralj R., Ferreira M.M.C., Basic validation procedures for regression models in QSAR and QSPR studies: theory and application, J. Braz Chem. Soc. 2009, 20, 770-787.

[47] Mardia K.V., Kent J.T., Bibbly J.M., Multivariate Analysis, Academic Press, New York, 1979.

[48] Sobańska A.W., Żydek G., Włodno P., Brzezińska E., Comparative (Q)SAR analysis of benzodiazepine derivatives with different biological activity, Eur. J. Med. Chem. 2015, 89, 147-155.

[49] Lipiński C.A., Lead- and drug-like compounds: the rule-of-five revolution, Drug Disc. Today: Technol. 2004, 1, 337-341

[50] Sobańska A.W., Application of planar chromatographic descriptors to the prediction of physicochemical properties and biological activity of compounds, J. Liq. Chromatogr. Rel. Technol. 2018, 41, 255-271.

Supplemental Material: The online version of this article offers supplementary material (https://doi.org/10.1515/chem-2019-0005). 